\title{
Buckling Mode Jump at Very Close Load Values in Unattached Flat-End Columns: Theory and Experiment
}

\author{
H. Kalathur ${ }^{1}$, T. Hoang ${ }^{1}$, R. S. Lakes ${ }^{1,2}$, W. J. Drugan ${ }^{1,3}$ \\ Adapted from J. Appl. Mech. 81(4), 041010 Sept. (2013). \\ ${ }^{1}$ Engineering Physics Department, Engineering Mechanics Program \\ University of Wisconsin-Madison, Madison, WI, 53706 \\ E-mail: ${ }^{2}$ lakes@engr.wisc.edu,${ }^{3} \underline{\text { drugan@engr.wisc.edu }}$
}

\begin{abstract}
Buckling of compressed flat-end columns loaded by unattached flat platens is shown, theoretically and experimentally, to occur first at the critical load and associated mode shape of a built-in column, followed extremely closely by a second critical load and different mode shape characterized by column end-tilt. The theoretical critical load for secondary or end tilt buckling is shown to be only $0.13 \%$ greater than the critical load for primary buckling, in which the ends are in full contact with the compression platens. The experimental value is consistent with this theoretical one. Interestingly, under displacement control, the first buckling instability is characterized by a smoothly-increasing applied load, whereas the closely-following second instability causes an abrupt and large load drop (and hence exhibits incremental negative stiffness). The end tilt buckling gives rise to large hysteresis that can be useful in structural damping but that is non-conservative and potentially catastrophic in the context of design of structural support columns.
\end{abstract}

\section{Introduction}

Post-buckling behavior is of interest in the context of design of new structures, assessment of existing ones, and, from a different perspective, in the context of hysteresis damping that can occur in post-buckled structures. In structures, the nature of the post-buckling behavior determines whether the structural member continues to carry load after buckling, in which case buckling may be tolerable, or whether load decreases after buckling, in which case buckling may be catastrophic.

As for damping, buckling can give rise to negative structural stiffness and consequently to high damping. For example, structures containing buckled flexible tubes exhibited negative stiffness and consequent giant increases in damping under small deformation oscillatory load (Lakes [1]). A combination of stiffness and viscoelastic damping is desirable for applications such as reduction of noise and vibration in machinery and vehicles. Structural materials, such as steel, brass and aluminum alloys, are stiff but have low damping. Polymer damping layers have higher damping, but they are not stiff; they are also highly temperature dependent. Structural damping based on negative stiffness is advantageous in that high damping and high stiffness can be achieved without the need for special materials that are temperature-dependent. High damping in composite structures or materials that contain negative stiffness elements arises from amplification of motion of interfaces (Lakes [1]).

To illustrate the notion of a negative-stiffness composite, consider a set of linear elastic springs having positive stiffness as shown in Figure 1. In the unstretched configuration, Figure 1(a), the force displacement relationship has positive slope, hence positive stiffness. Negative stiffness can be attained by first displacing the point $\mathrm{A}$ to the right, compressing the springs to reach the configuration shown in Figure 1(b). This configuration exhibits negative stiffness, and if displacement $u$ is controlled and if stiffness $k_{2}$ is sufficiently large, the negative stiffness is stable. If, however, the stiffness $k_{2}$ is small, the configuration is unstable, as a slight perturbation would cause the structure to snap-through, as shown in Figure 1(c). 


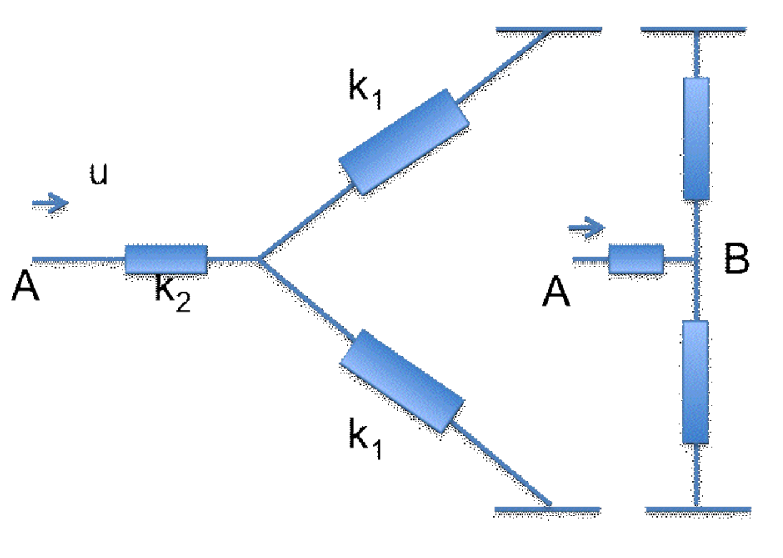

(a)

(b)

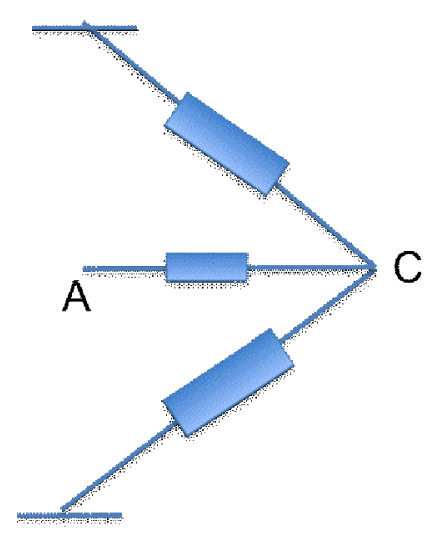

(c)

Figure 1: Geometry of linear elastic springs demonstrating negative stiffness in a lumped system. (Adapted from Jaglinski et al. [2]) Displacement $\mathrm{u}$ is applied at point A at left, producing a force.

(a) The springs are initially unstretched.

(b) The springs are deformed to a new equilibrium configuration that exhibits negative stiffness.

(c) Snap-through to a new stable configuration.

For structural damping, column buckling offers the advantage of much greater stiffness in comparison with buckling of tubes (which must be flexible owing to large local strain) or snap-through of discrete spring structures. Column post-buckling and snap back via end tilt has been used to demonstrate extremely high values of hysteresis damping in polymer based modules (Dong and Lakes [3]), in stiff modules based on steel (Dong and Lakes [4]), and in small amplitude oscillations (Kalathur and Lakes [5]).

The present research is directed to developing a clearer understanding of the process of postbuckling for columns, in particular, the interesting case in which the column ends are flat and not attached to the flat loading device. We show experimentally that this leads to a jumping instability between extremely closely spaced modes, which we also successfully model theoretically.

\section{Experiments}

\subsection{Methods}

Commercial, extruded PMMA rod (McMaster-Carr, \#8531K13) was used for the purpose of this work. Columns with length of about $200 \mathrm{~mm}$ were cut using a band saw (Pro-Tech 3203). The column ends were ground using $600 \mathrm{C}$ grade sanding paper to ensure flat surfaces. A digital caliper was used to determine the lengths and a digital micrometer to determine the diameter of the columns, about 6.453 $\mathrm{mm}$. Columns of different length were cut to obtain a range of slenderness ratio. The most slender columns had length $1=197.6 \mathrm{~mm}$.

Force-displacement relationships were obtained using a $90 \mathrm{kN}$ capacity servo-hydraulic test frame (MTS corp. Minneapolis, MN) calibrated to meet 1\% accuracy via ASTM specifications, and a digital oscilloscope (Tektronix, TDS 3012, 2 channel, 100MHz, 1.25GS/s) at ambient temperature. The digital oscilloscope was used to examine the Lissajous figure, that is, the force-displacement plot. The column was secured between the platens by moving the grips on the servo- hydraulic frame towards each other until contact. This caused a slight pre-load (about $9-18 \mathrm{~N}$ ) to be applied on the column to achieve proper contact. An amplified scale of $9 \mathrm{kN}$ maximum was used to improve resolution of force. The noise level in the load channel of the test frame was equivalent to $\pm 5.8 \mathrm{~N}$. For buckling threshold tests, the test frame was operated under displacement control with a $30 \mathrm{~s}$ ramp input. Since the 
emphasis in this experiment was the end condition behavior of the column, the amplitude of the ramp was sufficient that snap-through buckling occurs. While the main focus of the experiments was column behavior under unfastened end contact, so that the ends were free to tilt, another set of experiments was conducted with glued ends to achieve a built-in boundary condition for comparison purposes. Selected curves were fit using MATLAB. For hysteresis studies, displacement control was also used; the input waveform was sinusoidal in time.

\subsection{Experimental Results and Discussion}

The initial column shape and the buckled shapes of the column are shown in Figure 2. As compressive deformation increases, the column initially remains straight (Fig. 2(a)) and load increases linearly with the imposed displacement. When a critical imposed displacement is attained, initial buckling occurs with the ends remaining horizontal and in full contact with the platens, as shown in Fig. 2(b). Following a very slight additional imposed displacement, the buckling mode jumps to column end tilt buckling as shown in Fig. 2(c); a close-up image of the end tilt is shown in Fig. 2(d). The same sequence of buckling mode jump was observed for columns of slenderness $\mathrm{d} / \mathbf{l}$ from 0.033 to 0.11 . The edge contact gives rise to observable deformation of the column end as shown on the left in Fig. 2(d).

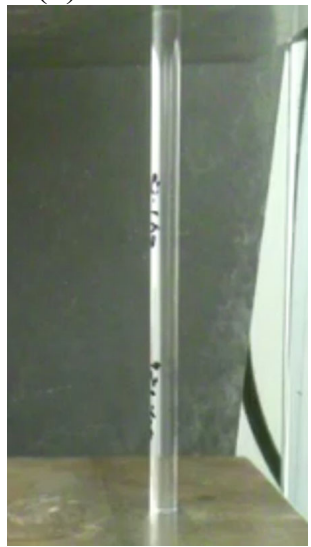

(a)

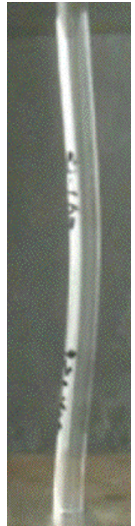

(b)

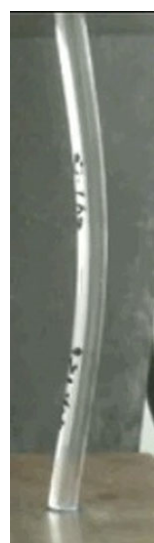

(c)

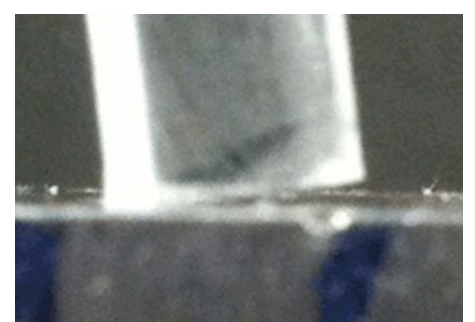

(d)

Figure 2: Polymer column. (a) Column secured between flat platens solely by contact under compression. (b) Column deflection at first buckling, identical to that of a clamped-ended column. (c) Column deflection at second buckling, exhibiting end tilt. (d) Column end tilt in close-up.

Figure 3(a) shows the force-displacement relationship of a column subject to a 30s ramp input. At first buckling, there is a change in slope of the curve, then there is an abrupt drop in load magnitude when the mode shifts to end tilt buckling. This happens when the lateral deflection (Fig. 2(b), (c)) exceeds the column diameter. Results from a curve fit for determining the buckle and snap thresholds (i.e., those of first and second buckling) are shown in Fig. 3(b). The lateral deflection versus displacement is shown in Figure 3(c) for further comparison with theory.

The criterion for first buckling was considered as a $5.8 \mathrm{~N}(2.3 \%)$ deviation of the curve fit from experimental data (Figure 3(b)) from the linear fit. This is based on the $\pm 5.8 \mathrm{~N}$ noise level (Figure $3(\mathrm{a}))$ in the load channel of the test frame.

For the most slender PMMA column (with $d=6.453 \mathrm{~mm}$ and $1=197.6 \mathrm{~mm}$ ), the experimental results show that the critical load (threshold) for tilt (second) buckling exceeds that for initial buckling by about $0.2 \%$. The critical load is obtained to greater precision than the load as a function of displacement because the curve fitting procedure averages the noise which is $\pm 5.8 \mathrm{~N}$ or $\pm 2.3 \%$. The $0.2 \%$ precision does not entail a corresponding accuracy, so, in view of the noise, the difference in buckling load calculated from experiment is regarded as consistent with theory (below) which predicts a $0.13 \%$ difference in buckling threshold. Moreover, the local deformation at the column edge shown 
in Figure 2(d) is not incorporated in the theory; this will give rise to a deviation that is difficult to quantify.

Figure 3(c) was obtained by taking individual frames from a video of a column with $d=6.453 \mathrm{~mm}$ and $1=150.4 \mathrm{~mm}$ subject to a $30 \mathrm{~s}$ ramp function. The individual frames were printed out on a sheet of paper and the respective lateral deflections were measured using a caliper. The measured values were carefully converted to give actual deflections. Since the measured values entail some degree of error due to resolution limitations, the standard deviation of all the measurements was determined and employed to obtain the lateral deflection. The error bars shown are based on the maximum deviation from the mean value of each of the obtained values of lateral deflection, which is $13 \%$.

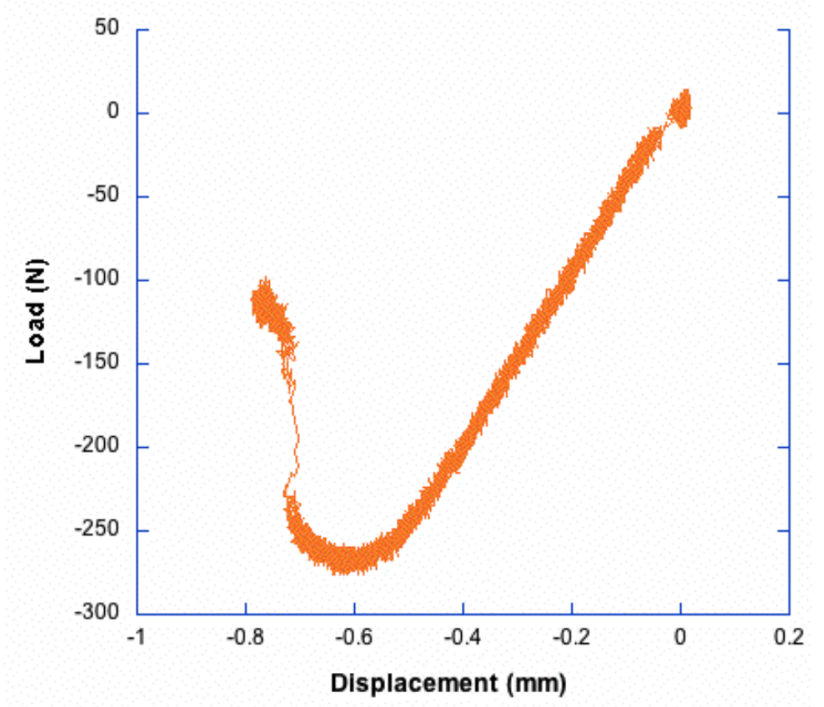

(a)

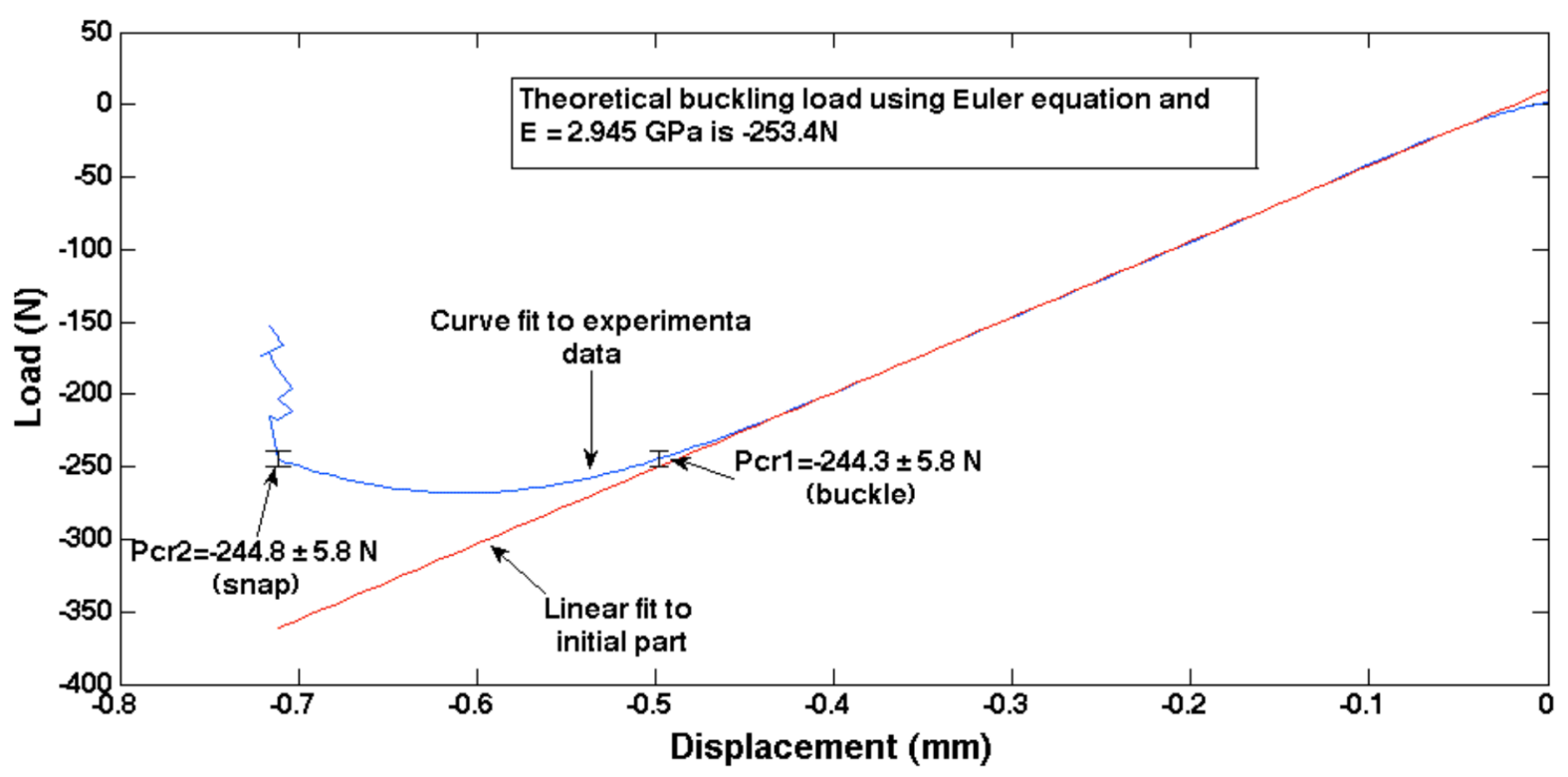

(b) 


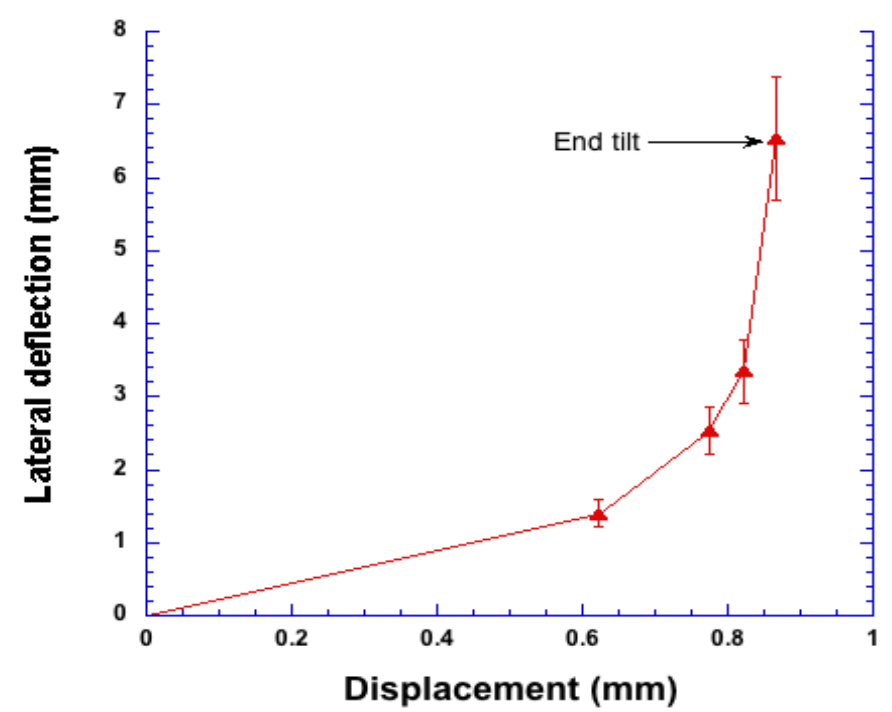

(c)

Figure 3: (a) The force-displacement relationship of a PMMA column with $d=6.453 \mathrm{~mm}$ and $\quad 1=$ 197.6mm from zero load through both buckling events.

(b) Curve fit of the full experimental data set but only up to just prior to snap (second buckling) instability. A linear fit to the initial part of the experimental data was used to determine the thresholds for initial buckling and end tilt buckling. On the left, a portion of the raw experimental data for snapthrough associated with tilt of column ends is shown.

(c) Lateral deflection vs. axial displacement of a column with $d=6.453 \mathrm{~mm}$ and $1=150.4 \mathrm{~mm}$.

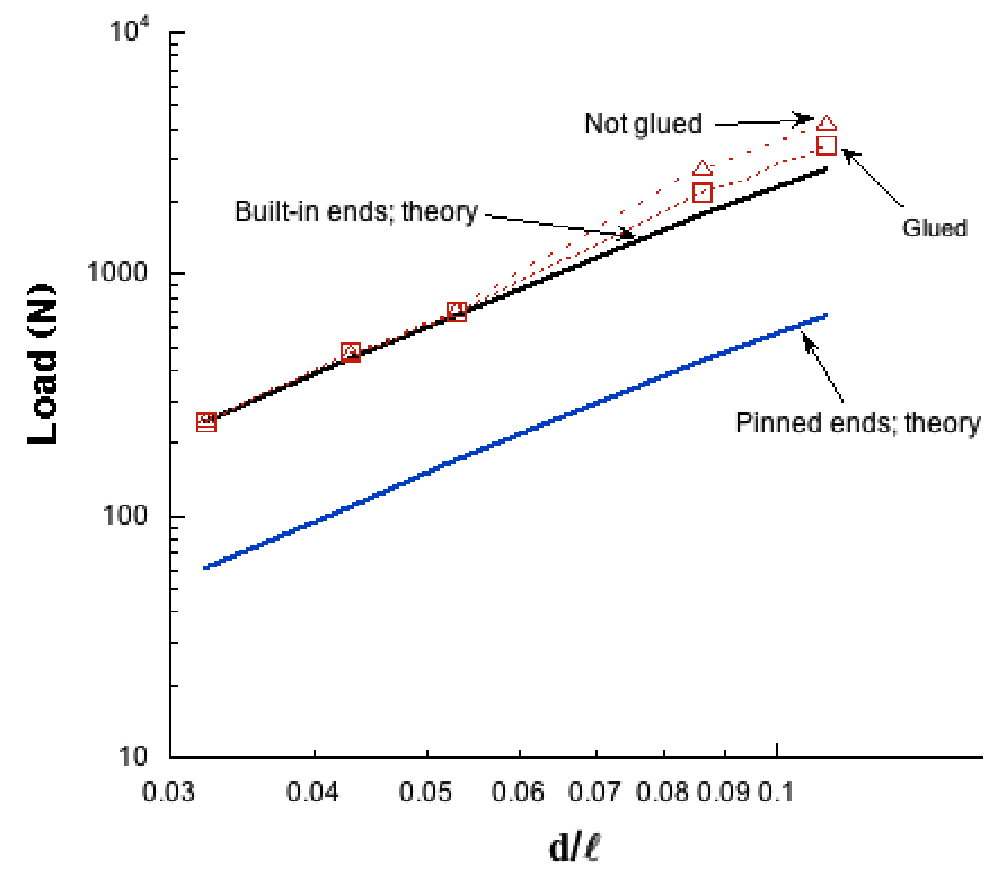

Figure 4: Load thresholds for buckling of PMMA columns for several aspect ratios; comparison of theory and experiment. Glued columns are considered as built-in.

As deformation is increased, the buckling mode jumps from a built in type mode to an end tilt 
mode in compressed unattached columns of slenderness $\mathrm{d} / 1$ from 0.033 to 0.11 ; theory (below) predicts this behavior. Buckling load was compared with theoretical predictions assuming a built-in end condition (here) and unattached flat ends (below). The Young's modulus input to the analysis was obtained from the slope of initial portion of the curve of load vs. deformation. Because PMMA is a viscoelastic material, the modulus depends on time. Therefore the slope was obtained at a time in the ramp function approaching that of the initial buckling. Figure 4 shows that for a slender column with $\mathrm{d} / \mathrm{l}=0.033$, the thresholds for not glued (free to end-tilt) and glued (built in) end conditions differ little and they agree very well with theory. The theoretical buckling load for the case of clamped ends and for $\mathrm{d} / 1=0.033$ was determined to be $253.4 \mathrm{~N}$ (Figure 4) based on the measured Young's modulus $\mathrm{E}=$ $2.95 \mathrm{GPa}$. The experimental result is $244.3 \mathrm{~N}$, a $3.6 \%$ difference.

Error analysis is as follows. Noise in the load channel is $\pm 5.8 \mathrm{~N}$ or $\pm 2.3 \%$. Thicker specimens would improve the ratio of signal to noise, but the load frame would not accommodate the requisite length of such specimens. There is also an uncertainty in the calculated buckling load due to the fact the commercial plastic rod is not perfectly round. Diameter variation by $0.62 \%$ gives rise to a $2.5 \%$ variance in area moment of inertia, hence in buckling load. Moreover the load frame calibration is to within $\pm 1 \%$. The maximum error is the sum of these, or $5.8 \%$; the root mean square (probable) error is $3.6 \%$. Therefore experiment and theory are considered to be in quite satisfactory agreement. We remark that neither the uncertainty in diameter nor in force sensitivity of the load frame contributes to the comparison between initial and final buckling because both loads are equally affected.

Moreover, the edges deform observably (Figure 2(d)). At higher $d / 1$, that is, for shorter columns of the same thickness, that deformation is more pronounced. Examination of the specimens after testing revealed localized yielding. This is the likely cause of the deviation of experiment from theory for the thicker specimens. The theory assumes a slender, perfectly cylindrical column, ignores shear, and does not incorporate local deformation of the ends. Therefore an increasing deviation between load via theory and experiment for thicker columns is to be expected. Such effects are difficult to quantify, therefore no error bars are shown in Figure 4. Finally, viscoelasticity in the columns was in part accounted for by using the slope of the load deformation curve at strain, hence time, approaching but below the value pertinent to buckling; the theory is based on elastic behavior. The creep buckling threshold has been analyzed (e. g. [6]) but not in the context of the mode jump considered here.

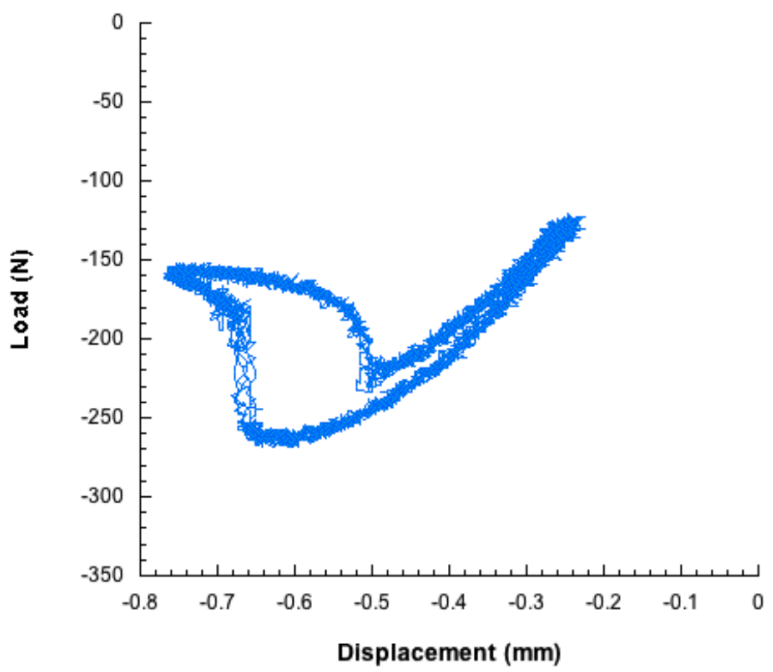

(a)

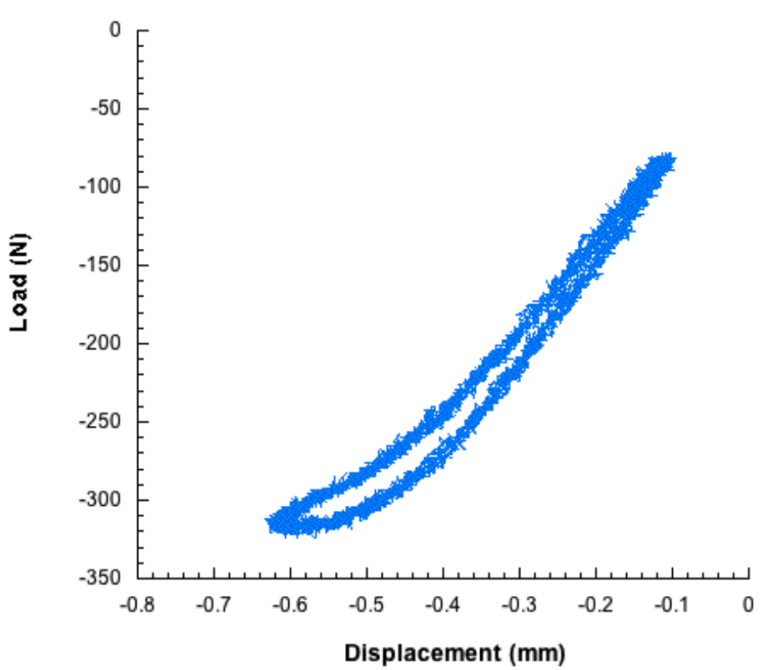

(b)

Figure 5: Hysteresis at $1 \mathrm{~Hz}$ of a PMMA column with $\mathrm{d} / \mathrm{l}=0.033$. The displacement amplitude is $0.53 \mathrm{~mm}$ and the column length is $197.6 \mathrm{~mm}$. (a) Ends unattached. (b) Ends glued. 
Figure 5(a) shows nonlinear hysteresis damping associated with end-tilt buckling and snap-back as deformation decreases. The energy loss is denoted by the area within the load-displacement curve. An effective damping $\Psi / 2 \pi$ (via specific damping capacity $\Psi$ ) of about 0.59 was achieved for the case of unattached ends free to tilt; this is far above the intrinsic linear damping for PMMA, which is about 0.07 at $1 \mathrm{~Hz}$. Damping can be enhanced further by restricting the range of displacement further to encompass only the hysteresis region. For comparison, the nonlinearity associated with post-buckling of a column with built-in (glued) ends (Figure 5 (b)) gives rise to a damping of only about 0.15 . The Lissajous figures consist of an initial linear portion, which refers to the initial stiffness of PMMA. The linear portion does not pass through zero due to initial applied displacement.

\section{Theoretical Modeling and Analysis}

We have successfully modeled theoretically the initial and secondary (end-tilt) buckling events observed in the experiments on compressed columns having flat, unattached ends. The initial buckling can be predicted by direct application of an analysis first done by Timoshenko and Gere [7] of buckling of a column having rounded ends; this is summarized in Section 3.1. The subsequent secondary buckling event can be analyzed in two different ways, leading to identical results; these analyses are presented in Sections 3.2 and 3.3.

\subsection{Timoshenko analysis of initial buckling of a column having rounded ends}

To facilitate theoretical treatment of the problem of initial buckling of a column with unattached flat ends resting against flat "rigid" platens which load it in compression, to explore the effect of end shape on buckling, and to provide the framework for one approach for analyzing the experimentallyobserved secondary buckling event, we model the column ends as being curved. We follow Timoshenko and Gere [7] and in two dimensions treat the ends as portions of a circle with radius $R$, as illustrated in Figure 6(a). When a column with rounded ends buckles laterally due to compressive loading by contact with rigid flat platens, the line of action of the compressive forces $P$ will displace a distance $b$ as shown Figure $6(\mathrm{a}, \mathrm{b})$. Since the angle of rotation of the ends of the bar is small, the displacement is $b=R \theta$.

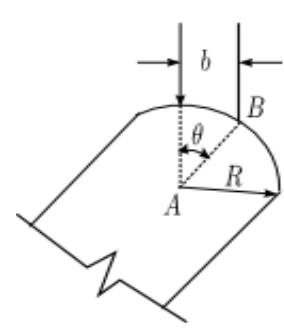

(a)

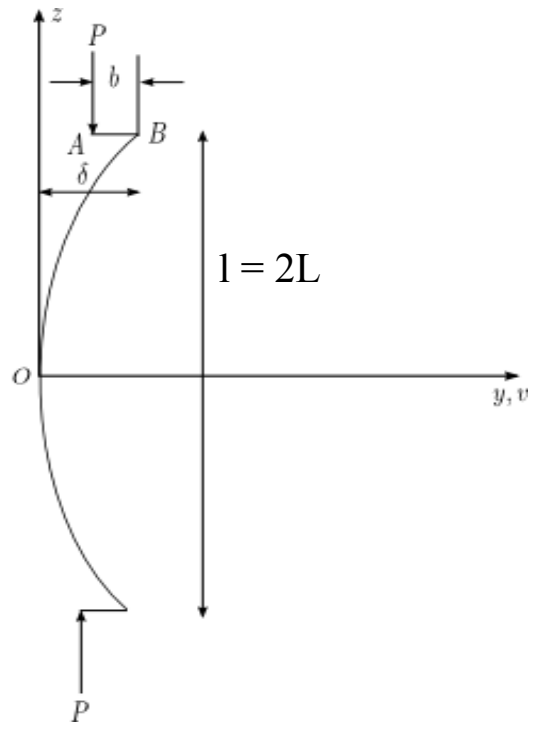

Figure 6. (a) Curved end of column, modeled as a portion of a circle having radius $R$;

(b) Displacement of forces action line due to rotation of curved column ends. 
To analyze the round-ended column, we treat half of a column since it first buckles into a symmetrical shape. We take the origin of the $y, z$ Cartesian coordinate system at the center $O_{\text {of }}$ the column as shown in Figure 6(b). We employ the slender beam moment-curvature relation:

$$
E I v^{\prime \prime}(z)=M(z)
$$

where $E, I, M(z)$ and $v(z)$ are Young's modulus, moment of inertia of the cross-section, moment and deflection at arbitrary cross-section location $z$, respectively. The moment is

$$
M(z)=P[\delta-v(z)-b]
$$

where $\delta=v(L)$ is the beam end deflection with respect to its center with $\mathrm{L}=1 / 2$.

Substituting (2) into (1) and differentiating the result twice with respect to $z$ gives:

$$
E I \frac{d^{4} v}{d z^{4}}+P \frac{d^{2} v}{d z^{2}}=0
$$

To solve (3) one needs four boundary conditions on the half-column; these are:

At $z=0$ : displacement and slope are zero:

$$
v(0)=v^{\prime}(0)=0 .
$$

At $z=L$ : shear force and moment are, respectively:

$$
E I v^{\prime \prime}(L)=-P v^{\prime}(L), \quad E I v^{\prime \prime}(L)=-P b=-P R v^{\prime}(L) .
$$

In the second of (5) we have employed the relation between deflection and slope $\nu^{\prime}=\tan \theta \approx \theta$ : $b=R \theta=R v^{\prime}(L)$. Nondimensionalizing (3), with dimensionless position $\xi=z / L$, we have

$$
\frac{d^{4} v}{d \xi^{4}}+k^{2} \frac{d^{2} v}{d \xi^{2}}=0
$$

where

$$
k^{2}=\frac{P L^{2}}{E I} .
$$

The boundary conditions then transform to:

$$
v(0)=v^{\prime}(0)=0, \quad v^{\prime \prime \prime}(1)=-k^{2} v^{\prime}(1), \quad \varepsilon v^{\prime \prime}(1)=-k^{2} v^{\prime}(1)
$$

where we have defined $\varepsilon=L / R$.

Equation (6) has the general solution

$$
v(\xi)=C_{1} \sin (k \xi)+C_{2} \cos (k \xi)+C_{3} \xi+C_{4},
$$

and application of (8) to (9) requires

$$
C_{1}=C_{3}=0, \quad C_{2}=-C_{4}, \quad C_{2} k^{2}(k \sin k+\varepsilon \cos k)=0,
$$

showing that the system has nontrivial solutions only if

$$
k \sin k+\varepsilon \cos k=0 .
$$

Equation (11), recalling the definition (7), summarizes the influence of end-roundedness on the initial buckling load. The case of interest here, to predict first buckling of the unattached flat-ended column, is the extreme one in which the column ends are perfectly flat: this occurs when $R \rightarrow \infty$, so that $\varepsilon=L / R \rightarrow 0$, showing that (11) reduces to, for the lowest buckling load and by use of (7): 


$$
\sin k=0 \Rightarrow k^{2}=\frac{P L^{2}}{E I}=\pi^{2} \Rightarrow P_{c r 1}=\frac{\pi^{2} E I}{L^{2}} .
$$

Thus, the initial buckling load for an unattached flat-ended column is the same as for a clamped-ended one. These results were obtained by Timoshenko and Gere [7].

We conclude that as the compressive load increases from zero to the (first) buckling load $P_{c r 1}=\frac{\pi^{2} E I}{L^{2}}$, the straight column buckles to the curved shape, from (9) with (10)

$$
v(\xi)=C_{4}[1-\cos (\pi \xi)]=f[1-\cos (\pi \xi)]
$$

having renamed $C_{4}$ as $f$. The results (12) and (13) clearly agree with the experimental results for the initial buckling load and column shape at first buckling described in Section 2.

\subsection{Analysis of secondary buckling via the Timoshenko formulation}

The static (or adjacent-equilibrium) method of stability analysis employed by Timoshenko and Gere [7] only gives the initial buckling load, not the behavior of the column after the load exceeds the critical value. In other words, it does not give the relation between the load $P$ and the maximum lateral column deflection $f\left(v_{\max }=2 f\right)$ from (13); the value of $f$ is not determined. Post-buckling theory for elastic structures deals with this situation and does give this information, e.g., Budiansky [8]. For a truly clamped ended column, the post-buckling curve (13) is always stable and the relation between the coefficient $f$ and the applied load level exceeding the initial buckling load is

$$
\frac{P}{P_{c r 1}}=1+\frac{1}{8} \frac{\pi^{2} f^{2}}{L^{2}}
$$

where $P_{c r 1}$ is that given in (12).

Here we show how (14) is derived in a simple, transparent way, applied to a column using the large deflection theory of a beam, Bolotin [9]; we show this because we shall employ this approach in our derivation of the second buckling load below.

The full moment-curvature relation for large deflections is:

$$
\frac{1}{\rho}=\frac{\frac{d^{2} v}{d z^{2}}}{\sqrt{1-\left(\frac{d v}{d z}\right)^{2}}}=\frac{M}{E I} .
$$

When deflections are small, this of course reduces to (1) to leading order. For a more accurate result, we employ the following expansion, valid for small $\frac{d v}{d z}$ :

$$
\left[1-\left(\frac{d v}{d z}\right)^{2}\right]^{-1 / 2}=1+\frac{1}{2}\left(\frac{d v}{d z}\right)^{2}+\frac{3}{8}\left(\frac{d v}{d z}\right)^{4}+\ldots
$$

Substitution of this and (2) into (15) gives 


$$
\frac{d^{2} v}{d z^{2}}\left[1+\frac{1}{2}\left(\frac{d v}{d z}\right)^{2}+\frac{3}{8}\left(\frac{d v}{d z}\right)^{4}+\ldots\right]+\frac{P v}{E I}+\frac{P(b-\delta)}{E I}=0
$$

Following Galerkin's method, we substitute (13) into the left-hand side of (17) and require the resulting equation to be orthogonal to the selected function $\cos (\pi \xi)=\cos \left(\frac{\pi z}{L}\right)$, Bolotin [9]:

$$
\int_{0}^{L}\left\{\frac{d^{2} v}{d z^{2}}\left[1+\frac{1}{2}\left(\frac{d v}{d z}\right)^{2}+\frac{3}{8}\left(\frac{d v}{d z}\right)^{1}+\ldots\right]+\frac{P v}{E I}+\frac{P(b-\delta)}{E I}\right\} \cos \left(\frac{\pi z}{L}\right) d z=0 .
$$

After integration, (18) reduces to

$$
\frac{P}{P_{c r 1}}=1+\frac{1}{8} \frac{\pi^{2} f^{2}}{L^{2}}+\frac{3}{64} \frac{\pi^{4} f^{4}}{L^{4}}+\ldots
$$

where $P_{c r 1}$ is that given in (12). Comparison with the exact solution of the same problem (elastica problem) using elliptic integrals shows that approximation (14) is accurate if $P<1.045 P_{c r 1}$ (Bolotin [9]).

Although the unattached flat-ended column undergoes initial buckling at the same critical load as the clamped-ended one, their post-buckling behaviors are entirely different. While the post-buckling shape (13) of the clamped ended column is stable, Budiansky [8], that of the unattached flat-ended column is not, as shown by the experiments described in Section 2 above. As described there, as the load is increased beyond the initial buckling load (12) for the unattached flat-ended column to a second, slightly higher critical load, the shape (13) having zero slopes at both column ends buckles into an entirely different shape having nonzero column end slopes, in which the column ends have rocked up onto their corners; this transition is illustrated in Figures 2 (b, c).

We model theoretically this change from the first buckled shape to the different second one by now treating the column ends as deviating very slightly from perfect flatness, i.e., as having a small curvature. This means $\varepsilon=L / R$ is not identically zero but is extremely small.

To determine the critical load for the second buckling event, we interpret the experimental observations in the context of a column whose ends have very small curvature: in this case, the column ends rocking onto their corners corresponds to the load application point moving from the center of the column end all the way to its corner, i.e., $b$ of Figure 6 going from zero to $b=d / 2$. The second critical buckling load is thus the applied load value needed for $b=d / 2$.

Assuming small $\varepsilon$, we expand $k$ in $\varepsilon$ through first order:

$$
k=k_{0}+k_{1} \varepsilon \text {. }
$$

Substituting (20) into (11) [which, recall, is valid for any end curvature] and retaining terms through first order of $\varepsilon$ gives

$$
k_{0} \sin \left(k_{0}\right)+\left[\cos \left(k_{0}\right)+k_{1} \sin \left(k_{0}\right)+k_{0} k_{1} \cos \left(k_{0}\right)\right]=0
$$

Enforcing this for each of the two $\varepsilon$ powers appearing shows that

$$
k_{0}=\pi, \quad k_{1}=-\frac{1}{\pi} .
$$

Similarly to (20), we expand the other needed variables in $\varepsilon$ through first order:

$$
f=f_{0}+f_{1} \varepsilon
$$




$$
P=P_{0}+P_{1} \varepsilon
$$

Employing (20) and (23) in (13) and writing the result through first order in $\varepsilon$ gives:

$$
v(\xi)=f_{0}\left(1-\cos \left[k_{0} \xi\right]\right)+\left(f_{1}-f_{1} \cos \left[k_{0} \xi\right]+f_{0} k_{1} \xi \sin \left[k_{0} \xi\right]\right) \varepsilon .
$$

Similarly to (18), we impose the condition

$$
\int_{0}^{L}\left\{\frac{d^{2} v}{d z^{2}}\left[1+\frac{1}{2}\left(\frac{d v}{d z}\right)^{2}+\frac{3}{8}\left(\frac{d v}{d z}\right)^{1}+\ldots\right]+\frac{P v}{E I}+\frac{P(b-\delta)}{E I}\right\} \cos \left[\left(k_{0}+k_{1} \varepsilon\right) \frac{z}{L}\right] d z=0 .
$$

We obtain from this after integration and simplification the relation between load and deflection amplitude

$$
\frac{P_{0}}{P_{c r 1}}=1+\frac{1}{8} \frac{\pi^{2} f_{0}^{2}}{L^{2}}
$$

The displacement of the force is, from $b=R \theta=R v^{\prime}(L)$ :

$$
b=\frac{R}{L} \nu^{\prime}(1)=\frac{1}{\varepsilon} \nu^{\prime}(1)=f_{0} k_{0} k_{1} \cos \left[k_{0}\right]=f_{0} .
$$

Hence when $b=d / 2$

$$
v_{\max }=\delta=v(1)=2 f_{0}+2 f_{1} \varepsilon \approx 2 f_{0}=2 b=d .
$$

Thus, the second critical load - the load at which the load application point has just reached the corner of the column - is:

$$
\frac{P_{c r 2}}{P_{c r 1}} \approx \frac{P_{0}}{P_{c r 1}}=1+\frac{\pi^{2}}{8}\left(\frac{d}{2 L}\right)^{2}
$$

Employing the experimental data from Section 2:

$$
d=6.453 \mathrm{~mm}, \quad ?=2 L=197.6 \mathrm{~mm}, \text { we calculate from }(30): \frac{P_{c r 2}-P_{c r 1}}{P_{c r 1}}=0.13 \% \text {. }
$$

That is, the second critical load is predicted to be $0.13 \%$ higher than the first critical load. Equation (29) provides a useful result for comparing with the experiments: it shows that when the maximum lateral deflection of the column reaches approximately the column diameter, the change in buckled patterns occurs. As noted in Section 2, our experiments were in accord with this prediction.

\subsection{Alternative analysis of secondary buckling load}

We now present a completely different analysis of the secondary buckling load which leads to exactly the same result derived in the previous section. The approach here is to consider two identical columns under unchanging end conditions: one column has built-in ends and is loaded above its initial buckling load, and the other has flat ends and is loaded by compressive loads acting at one corner of each end and is deformed such that the end has rotated onto the corner, both as shown in Figure 7 . The 
secondary buckling load is the load value at which the vertical deflections of the two columns first becomes identical.

When $P>P_{c r 1}$, the post-buckled shape of the clamped-ended column is that obtained in (13) and the vertical deflection $\Delta$ in Figure 7(a) is obtained as in, e.g. Budiansky [8]:

$$
\Delta=\int_{0}^{L} \frac{1}{2}\left[v^{\prime}\left(z_{0}\right)\right]^{2} d z_{0}=\frac{1}{2 L} \int_{0}^{1}\left[v^{\prime}(\xi)\right]^{2} d \xi=\frac{\pi^{2} f^{2}}{4 L} .
$$

Thus, combining (14) and (31) gives the relation between the applied load and the vertical deflection:

$$
\frac{P}{P_{\text {cr } r}}=1+\frac{\Delta}{2 L} \text {. }
$$

Next consider a column compressed by forces applied at the corners in the manner illustrated in Figure 7(b). The boundary conditions in (8) do not change except for the moment, which becomes $M=-P d / 2$, so these conditions become:

$$
v(0)=v^{\prime}(0)=0, \quad v^{\prime \prime \prime}(1)=-k^{2} v^{\prime}(1), \quad v^{\prime \prime}(1)=-k^{2} d / 2 .
$$

Differential equation (6) with boundary conditions (33) has solution:

$$
v(\xi)=\frac{d}{2 \cos (k)}[\cos (k \xi)-1]
$$

The vertical deflection corresponding to the curve (34) as illustrated in Figure 7(b) is obtained by substitution of (34) into (31):

$$
\frac{\Delta}{L}=\frac{d^{2}}{16 L^{2}} \frac{k}{\cos ^{2}(k)}[k-\sin (k) \cos (k)] .
$$

The second critical load, $P_{c r 2}$, producing the transition shown in Figure 2(b) to 2(c) is the applied load level at which the vertical deflection in the column of Figure 7(a) just equals that of the column of Figure 7(b); this means that the $\Delta$ in (35) just equals that in (32). Thus, substitution of (35) into (32) and using (12) to express $P / P_{c r 1}$ in terms of $k$ gives the following equation for $k$ :

$$
\frac{k^{2}}{\pi^{2}}=1+\frac{d^{2}}{32 L^{2}} \frac{k}{\cos ^{2}(k)}[k-\sin (k) \cos (k)]
$$

If we call the smallest positive $k$ solution to (36) $k_{c r}$, then the second critical buckling load $P_{c r 2}$ is given by:

$$
\frac{P_{c r 2}}{P_{c r 1}}=\frac{k_{c r}^{2}}{\pi^{2}}
$$

It is easy to verify numerically that (37) agrees with (30). Alternatively, if we assume $\left(\frac{d}{2 L}\right)^{2}<<1$, solution of (36) for $\frac{k_{c r}^{2}}{\pi^{2}}$ through order $\left(\frac{d}{2 L}\right)^{2}$ gives exactly the right side of (30). 


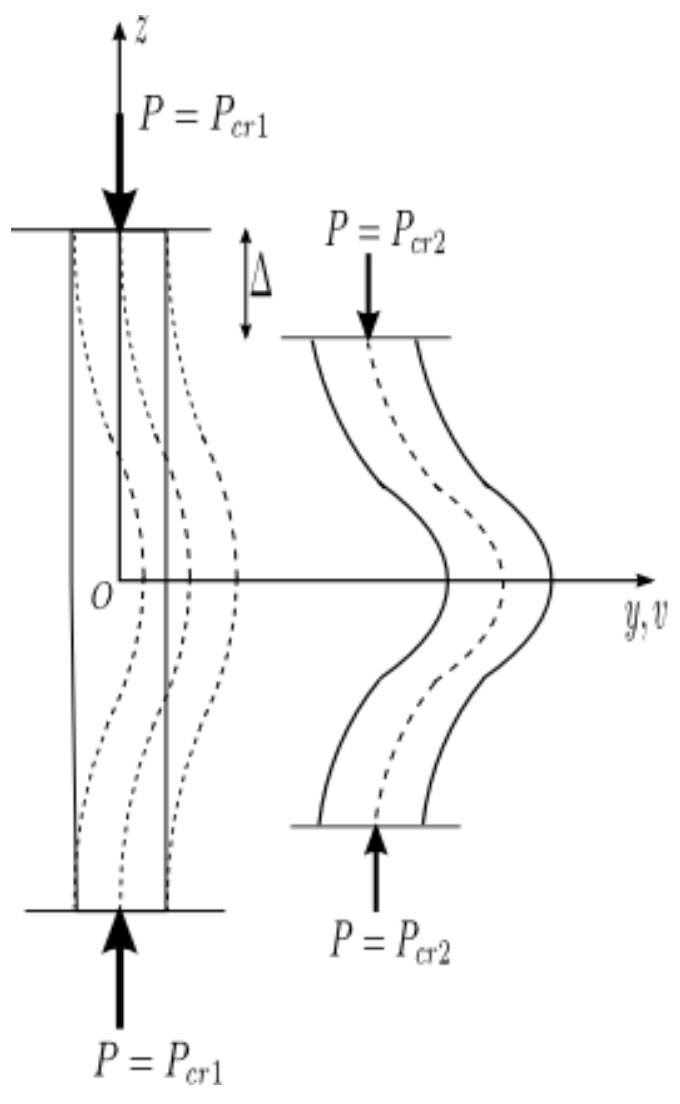

(a)

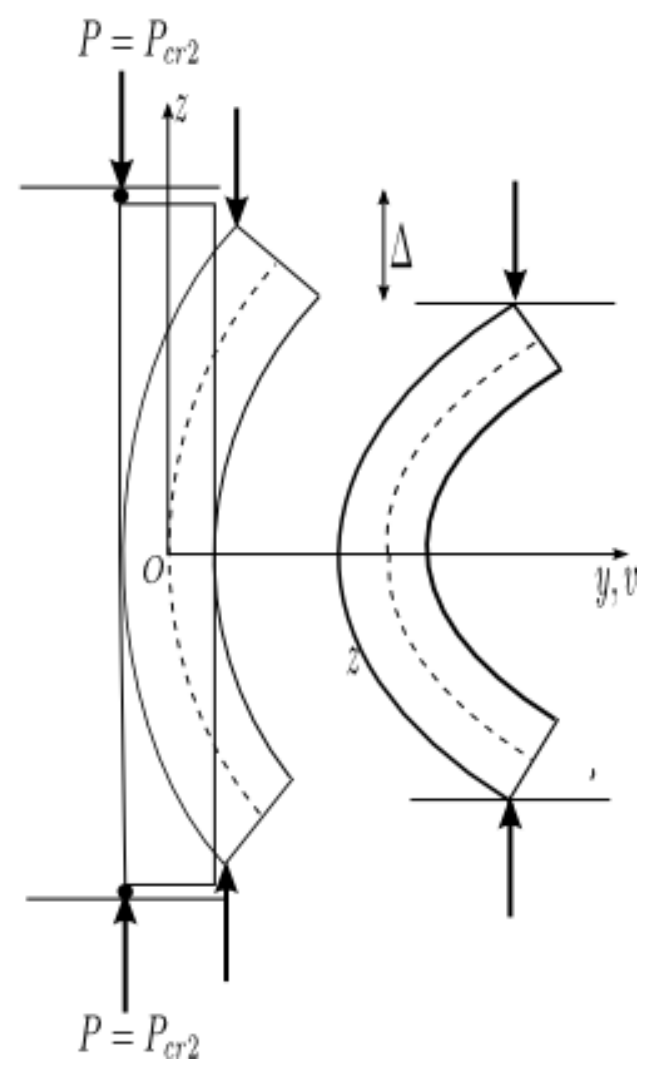

(b)

Figure 7. The two columns with different end conditions analyzed to predict the second buckling load.

\section{Discussion and Conclusions}

Buckling mode jump from built in mode to end tilt mode, under an extremely small increase in applied load, was observed for compressed unattached flat-ended columns of slenderness $\mathrm{d} / \mathrm{l}$ from 0.033 to 0.11 . This behavior is consistent with theory, which predicts that secondary or end tilt buckling is only $0.13 \%$ greater than the threshold for primary buckling in which the ends are in full contact with the compression platens.

The load reduction during end tilt buckling is non-conservative in the context of design of structural support columns. Any slender column that is not fixed at the ends, either by intent as in a press-fit column, or from lack or degradation of fasteners, is subject to collapse under sufficient load. This is in contrast with pin-ended or built-in columns which carry load that gradually increases in the post-buckling regime. Indeed, columns are provided with a variety of end conditions in practice. For example, wood columns may be provided with hinge connections (American Wood Council [10]) in which the standard pin-end analysis is appropriate. Also, press-fit ends are used in which the flat end of a column is supported by a metal bearing plate and centered by a slender nail or pin through the center; in such a case there is minimal constraint on end rotation. This constraint would be further weakened by corrosion of the nail or pin with time, causing the end constraint to approach even more closely a pure press fit condition.

As for designed dampers, end tilt buckling gives rise to large hysteresis that can be useful in structural damping. In that context, the change in contact condition is critical in achieving high damping and consequently a high stiffness-damping product (Dong and Lakes [3], Kalathur and Lakes 
[5]). Dampers of this type are stabilized by incorporating press-fit columns designed to buckle in an assemblage of thicker built-in columns that remain straight under a range of intended loads (Dong and Lakes [3]). Dong and Lakes [3] demonstrated that such a module design, in which the buckling columns are retained solely by preload, remains coherent and exhibits high hysteresis over many cycles of deformation. Dampers based on column post buckling with tilt of the ends have been made from steel (Dong and Lakes [4]); the initial stiffness corresponds to that of steel, and the maximum damping $\Psi / 2 \pi$ can be as large as 2 . Post-buckling of built-in columns, by contrast, exhibits softening of the slope of the load-deformation curve but no snap-through effect or large hysteresis [Figure 5(b)]; the damping in this case is solely due to the intrinsic viscoelasticity of the column material. Damping based on column post-buckling is nonlinear but that is not necessarily a disadvantage. High damping metals are used in their nonlinear regime to absorb high amplitude vibration; e.g. copper manganese alloys for naval ship propellers and industrial hammers; zinc aluminum alloys in engines and industrial hammers. Column-based stablized damper modules could enable better performance using common structural materials.

\section{References}

[1] Lakes, R. S., 2001, "Extreme damping in compliant composites with a negative stiffness phase," Philosophical Magazine Letters, 81(2), 95-100.

[2] Jaglinski, T., Stone, D. S., Lakes, R. S., 2005, "Internal friction study of a composite with a negative stiffness constituent," Journal of Materials Research, 20(9), 2523-2533.

[3] Dong, L., Lakes, R. S., 2012, "Advanced damper with negative structural stiffness elements," Smart Materials and Structures, 21, 075026.

[4] Dong, L. and Lakes, R. S., 2013, "Advanced damper with high stiffness and high hysteresis damping based on negative structural stiffness," Int. J. Solids Struct., 50, 2413-2423.

[5] Kalathur, H., Lakes, R. S., 2013 "Column dampers with negative stiffness: high damping at small amplitude," Smart Materials and Structures, accepted May, 2013.

[6] Vinogradov, A. M., 1987, "Buckling of Viscoelastic Beam Columns," AIAA journal, 26(3), 479483.

[7] Timoshenko, S. and Gere, J. M., 1963, Theory of Elastic Stability, McGraw-Hill.

[8] Budiansky, B., 1974, "Theory of Buckling and Post-Buckling Behavior of Elastic Structures," Advances in Applied Mechanics 14, 1-65.

[9] Bolotin, V., 1964, The Dynamic Stability of Elastic Systems, Holden-Day.

[10] American Wood Council, 2012, Engineered Wood Construction, Section M10, Mechanical Connections, 64, 74 . 